\title{
Spectral Properties of Nonhomogenous Differential Equations with Spectral Parameter in the Boundary Condition
}

\section{Özkan Karaman}

\author{
Abstract \\ In this paper, using the boundary properties of the analytic functions \\ we investigate the structure of the discrete spectrum of the boundary \\ value problem

$$
\begin{aligned}
i y_{1}^{\prime}+q_{1}(x) y_{2}-\lambda y_{1} & =\varphi_{1}(x) \\
-i y_{2}^{\prime}+q_{2}(x) y_{1}-\lambda y_{2} & =\varphi_{2}(x), \quad x \in R_{+}
\end{aligned}
$$ \\ and the condition

$$
\left(a_{1} \lambda+b_{1}\right) y_{2}(0, \lambda)-\left(a_{2} \lambda+b_{2}\right) y_{1}(0, \lambda)=0
$$ \\ where $q_{1}, q_{2}, \varphi_{1}, \varphi_{2}$ are complex valued functions, $a_{k} \neq 0, b_{k} \neq 0$, \\ $k=1,2$ are complex constants and $\lambda$ is a spectral parameter. In this \\ article, we investigate the spectral singularities and eigenvalues of (0.1), \\ $(0.2)$ using the boundary uniqueness theorems of analytic functions. In \\ particular, we prove that the boundary value problem $(0.1),(0.2)$ has a \\ finite number of spectral singularities and eigenvalues with finite multi- \\ plicities under the conditions,

$$
\begin{aligned}
& \sup _{x \in R_{+}}\left[\quad\left|\quad \varphi_{k}(x)\right| \exp \left(\varepsilon x^{\delta}\right)\right]<\infty, k=1.2 \\
& \sup _{x \in R_{+}}\left[\quad\left|\quad q_{k}(x)\right| \exp \left(\varepsilon x^{\delta}\right)\right]<\infty, k=1.2
\end{aligned}
$$ \\ for some $\varepsilon>0, \quad \frac{1}{2}<\delta<1$. \\ Key Words: Schrödinger equation, spectrum, spectral operators, singularity. \\ 2010 Mathematics Subject Classification: Primary 81Q05, 47A10; Secondary 47B40 \\ Received: February, 2013. \\ Revised: April, 2013. \\ Accepted: February, 2014.
} $34 \mathrm{~L} 05$. 


\section{Introduction}

Let us consider the boundary value problem the $L[\lambda]$ generated in Hilbert space of vector valued functions the $L^{2}\left(R_{+}, C_{2}\right)$ by the system

$$
\begin{aligned}
i y_{1}^{\prime}+q_{1}(x) y_{2} & =\lambda y_{1}, \\
-i y_{2}^{\prime}+q_{2}(x) y_{1} & =\lambda y_{2}, \quad x \in R_{+}
\end{aligned}
$$

and the spectral parameter dependent boundary condition

$$
\left(a_{1} \lambda+b_{1}\right) y_{2}(0, \lambda)-\left(a_{2} \lambda+b_{2}\right) y_{1}(0, \lambda)=0 .
$$

Under the condition

$$
\sup _{x \in R_{+}}\left[\left|q_{k}(x)\right| \exp \left(\varepsilon x^{1+\delta}\right)\right]<\infty, \delta>0, \quad k=1,2
$$

it is proved that the $L[\lambda]$ has finite number of eigenvalues and spectral singularities with finite multiplicities. Furthermore, the principal functions corresponding to the eigenvalues of the $L[\lambda]$ belong to the $L^{2}\left(R_{+}, C_{2}\right)$ and the principal functions corresponding to spectral singularities belong to a Hilbert space containing the $L^{2}\left(R_{+}, C_{2}\right)[9]$. We now consider the operator the $L_{1}(\lambda)$ generated in

$$
L^{2}\left(R_{+}, C_{2}\right):=\left\{f(x)=\left(\begin{array}{c}
f_{1}(x) \\
f_{2}(x)
\end{array}\right), \int_{0}^{\infty}\left\{\left|f_{1}(x)\right|^{2}+\left|f_{2}(x)\right|^{2}\right\} d x<\infty\right\}
$$

by the system

$$
\begin{aligned}
i y_{1}^{\prime}+q_{1}(x) y_{2}-\lambda y_{1} & =0, \\
-i y_{2}^{\prime}+q_{2}(x) y_{1}-\lambda y_{2} & =0, \quad x \in R_{+}
\end{aligned}
$$

and the spectral parameter-dependent boundary condition

$$
\left(a_{1} \lambda+b_{1}\right) y_{2}(0, \lambda)-\left(a_{2} \lambda+b_{2}\right) y_{1}(0, \lambda)=0
$$

where $q_{k}, k=1,2$, are complex valued functions $\lambda$ is the spectral parameter, $\mathrm{a}_{k}, b_{k}$ are complex constants, $b_{k} \neq 0, k=1,2$. Moreover $\left|a_{1}\right|^{2}+\left|a_{2}\right|^{2} \neq 0$. Note that the spectral analysis of homogeneous Schrödinger, Strum-Liouville and Dirac equations with spectral singularities were studied in details in $[3-9]$. 


\section{The Solutions of Equation (1.1)}

Let us suppose that

$$
\left|q_{k}(x)\right| \leq c(1+x)^{-(1+\varepsilon)}, \quad k=1,2, \quad x \in R_{+}, \varepsilon>0
$$

holds, where $c>0$ is a constant. The following results were given in [1]. Under the condition (2.1), the equation (1.1) has the following vector solutions

$$
e^{+}(x, \lambda)=\left(\begin{array}{c}
e_{1}^{+}(x, \lambda) \\
e_{2}^{+}(x, \lambda)
\end{array}\right)=\left(\begin{array}{l}
\int_{0}^{\infty} H_{12}(x, t) e^{i \lambda t} d t \\
e^{i \lambda x}+\int_{0}^{\infty} H_{22}(x, t) e^{i \lambda t} d t
\end{array}\right)
$$

for $\lambda \in \overline{\mathbf{C}}_{+}$and

$$
e^{-}(x, \lambda)=\left(\begin{array}{c}
e_{1}^{-}(x, \lambda) \\
e_{2}^{-}(x, \lambda)
\end{array}\right)=\left(\begin{array}{l}
e^{-i \lambda x}+\int_{0}^{\infty} H_{11}(x, t) e^{-i \lambda t} d t \\
\int_{0}^{\infty} H_{21}(x, t) e^{-i \lambda t} d t
\end{array}\right)
$$

for $\lambda \in \overline{\mathbf{C}}_{-}$where

$$
\begin{aligned}
& C_{+}(x, \lambda)=\{\lambda: \lambda \in C, \operatorname{Im} \lambda \geq 0\} \\
& C_{-}(x, \lambda)=\{\lambda: \lambda \in C, \operatorname{Im} \lambda \leq 0\}
\end{aligned}
$$

moreover the kernels $H_{k j}(x, t), k, j=1,2$, satisfy the inequalities

$$
\left|H_{k j}(x, t)\right| \leq c \sum_{n=1}^{2}\left|q_{n}\left(\frac{x+t}{2}\right)\right|
$$

where $c>0$ is a constant. Therefore the functions $e_{k}^{+}(x, \lambda)$ and $e_{k}^{-}(x, \lambda)$ $k=1,2$ are analytic with respect to $\lambda$ in $\mathbf{C}_{+}, \mathbf{C}_{-}$and continuous on $\overline{\mathbf{C}}_{+}$ and $\overline{\mathbf{C}}_{-}$, respectively. Moreover $e^{+}(x, \lambda)$ and $e^{-}(x, \lambda)$ satisfy the following asymptotic equalities $([1])$

$$
e^{+}(x, \lambda)=\left(\begin{array}{l}
o(1) \\
e^{i \lambda x}+o(1)
\end{array}\right), \quad \lambda \in \overline{\mathbf{C}}_{+}, \quad \lambda \rightarrow \infty
$$

and

$$
e^{-}(x, \lambda)=\left(\begin{array}{l}
e^{-i \lambda x}+o(1) \\
o(1)
\end{array}\right), \quad \lambda \in \overline{\mathbf{C}}_{+}, \quad \lambda \rightarrow \infty .
$$


From (2.5) and (2.6) we have

$$
W\left\{e^{+}, e^{-}\right\}=\lim _{x \rightarrow \infty} W\left\{e^{+}(x, \lambda), e^{-}(x, \lambda)\right\}=-1
$$

for $\lambda \in R$, where $W\left\{y^{(1)}, y^{(2)}\right\}$ is the wronskian of the solutions of $y^{(1)}$ and $y^{(2)}$ which is defined as

$$
W\left\{y^{(1)}, y^{(2)}\right\}=y_{1}^{(1)} y_{2}^{(2)}-y_{1}^{(2)} y_{2}^{(1)},
$$

here $y^{(k)}=\left(\begin{array}{c}y_{1}^{(k)} \\ y_{2}^{(k)}\end{array}\right), \quad k=1,2$. Therefore $e^{+}(x, \lambda)$ and $e^{-}(x, \lambda)$ are the fundamental solutions of the system (1.1) for $\lambda \in \overline{\mathbf{C}}_{+}$. Now we will discuses the spectrum of $L(\lambda)$ defined as

$$
\begin{aligned}
i y_{1}^{\prime}+q_{1}(x) y_{2}-\lambda y_{1} & =\varphi_{1}(x) \\
-i y_{2}^{\prime}+q_{2}(x) y_{1}-\lambda y_{2} & =\varphi_{2}(x), \quad x \in R_{+}
\end{aligned}
$$

and the condition

$$
\left(a_{1} \lambda+b_{1}\right) y_{2}(0, \lambda)-\left(a_{2} \lambda+b_{2}\right) y_{1}(0, \lambda)=0
$$

where $\varphi(x)=\left(\begin{array}{l}\varphi_{1}(x) \\ \varphi_{2}(x)\end{array}\right)$ is complex valued functions. We proved that $L(\lambda)$ has a finite number of eigenvalues and spectral singularities under the conditions

$$
\sup _{x \in R_{+}}\left[\left|\varphi_{k}(x)\right| \exp \left(\varepsilon x^{\delta}\right)\right]<\infty, k=1.2 \quad \varepsilon>0, \quad \frac{1}{2}<\delta<1
$$

by using analytic continuation method [7].

\section{Eigen Values and Spectral Singularities of (2.8) and (2.9)}

From (2.5), (2.6) and (2.7) it is not difficult to see that

$$
\begin{aligned}
y(x, \lambda) & =i e^{-}(x, \lambda) I^{+}(x, \lambda)-i e^{+}(x, \lambda) I^{-}(x, \lambda) \\
& +a^{-}(\lambda) e^{+}(x, \lambda)-a^{+}(\lambda) e^{-}(x, \lambda),
\end{aligned}
$$

where $\lambda \in \overline{\mathbf{C}}_{+}$and

$$
\begin{aligned}
I^{ \pm}(x, \lambda) & =i \int_{x}^{\infty}\left(e_{1}^{ \pm}(t, \lambda) \varphi_{2}(t)+e_{2}^{ \pm}(t, \lambda) \varphi_{1}(t)\right) d t, \\
a^{ \pm}(\lambda) & =i I^{ \pm}(0, \lambda)+\left(a_{1} \lambda+b_{1}\right) e_{2}^{ \pm}(0, \lambda)-\left(a_{2} \lambda+b_{2}\right) e_{1}^{ \pm}(0, \lambda) .
\end{aligned}
$$

Now, we have the following lemma: 
Lemma 3.1. If (2.1) and (2.10) hold then

$$
\sigma_{d}(L)=\left\{\lambda: \quad \lambda \in \mathbf{C}_{+}, \quad a^{+}(\lambda)=0\right\}
$$

where $\sigma_{d}(L)$ denotes the set of eigenvalues of the equations (2.8).

Proof. Let $\lambda_{0} \in \mathbf{C}_{+}$. From (2.5) and (2.6) we get that $e^{+}\left(x, \lambda_{0}\right) \in L^{2}\left(R_{+}, C_{2}\right)$ and $e^{-}\left(x, \lambda_{0}\right) \notin L^{2}\left(R_{+}, C_{2}\right)$. Since

$$
i e^{-}\left(x, \lambda_{0}\right) I^{+}(x, \lambda)=o\left(\exp \left\{-\frac{\varepsilon}{2} x^{\delta}\right\}\right), \quad x \rightarrow \infty,
$$

and

$$
i e^{+}\left(x, \lambda_{0}\right) I^{-}(x, \lambda)=o\left(\exp \left\{-\frac{\varepsilon}{2} x^{\delta}\right\}\right), \quad x \rightarrow \infty,
$$

it follows from (3.1) that $y\left(x, \lambda_{0}\right)$ belongs to $L^{2}\left(R_{+}, C_{2}\right)$ if and only if $a^{+}(\lambda)=$ 0 . Analogously from (3.1), we have

$$
\sigma_{s s}(L)=\left\{\lambda: \quad \lambda \in R, \quad a^{+}(\lambda)=0\right\}
$$

where $\sigma_{s s}(L)$ denotes the set of spectral singularities of equations (2.8). It follows from (3.2) and (3.3) that in order to investigate the structure of the eigenvalues and the spectral singularities of equations (2.8), we need to discuss the structure of zeros of $a^{+}(\lambda)$ in $\overline{\mathbf{C}}_{+}$. In order to do so, we write the following:

$$
M_{1}=\left\{\lambda: \quad \lambda \in \mathbf{C}_{+}, \quad a^{+}(\lambda)=0\right\}, \quad M_{2}=\left\{\lambda: \quad \lambda \in R, \quad a^{+}(\lambda)=0\right\} .
$$

Similarly from (3.2) and (3.3), we see that

$$
\sigma_{d}(L)=\left\{\lambda: \quad \lambda \in M_{1}\right\}, \quad \sigma_{s s}(L)=\left\{\lambda: \quad \lambda \in M_{2}\right\} .
$$

Lemma 3.2. If (2.1) and (2.10) hold, then the following occurs. The set $M_{1}$ is bounded and has a countable number of elements, and its limit points can lie in a bounded subinterval of the real axis. The set $M_{2}$ is compact.

Proof. Using (2.2) and (2.10), we get that the function $a^{+}(\lambda)$ is analytic in $\mathbf{C}_{+}$, continuous in $\overline{\mathbf{C}}_{+}$, and

$$
\begin{array}{r}
a^{+}(\lambda)=b_{1}+\lambda\left\{a_{1}+a_{1} \int_{0}^{\infty} H_{22}(0, t) e^{i \lambda t}-a_{2} \int_{0}^{\infty} H_{12}(0, t) e^{i \lambda t} d t\right\} \\
+\int_{0}^{\infty} s^{*}(t) e^{i \lambda t} d t
\end{array}
$$


where

$$
\begin{aligned}
s^{*}(t)= & b_{1} H_{22}(0, t)-b_{2} H_{12}(0, t) \\
& +i\left\{\varphi_{1}(t)+\varphi_{2}(t) \int_{0}^{t} H_{12}(t, s) d s+\varphi_{1}(t) \int_{0}^{t} H_{22}(t, s) d s\right\}
\end{aligned}
$$

Applying integration by parts we find

$$
a^{+}(\lambda)=\lambda a_{1}+b_{1}+\int_{0}^{\infty} S^{+}(t) e^{i \lambda t} d t
$$

where

$$
\begin{aligned}
& \lambda a_{1} \int_{0}^{\infty} H_{22}(0, t) e^{i \lambda t} d t=A+i a_{1} \int_{0}^{\infty} \frac{d}{d t}\left(H_{22}(0, t)\right) e^{i \lambda t} d t \\
& \lambda a_{2} \int_{0}^{\infty} H_{12}(0, t) e^{i \lambda t} d t=B+i a_{2} \int_{0}^{\infty} \frac{d}{d t}\left(H_{12}(0, t)\right) e^{i \lambda t} d t
\end{aligned}
$$

and

$$
\begin{aligned}
S^{+}(t) & =\frac{d}{d t}\left(i a_{1} H_{22}(0, t)-i a_{2} H_{12}(0, t)\right)+b_{1} H_{22}(0, t) \\
& -b_{2} H_{12}(0, t)+i\left\{\varphi_{1}(t)+\varphi_{2}(t) \int_{0}^{t} H_{12}(t, s) d s+\varphi_{1}(t) \int_{0}^{t} H_{22}(t, s) d s\right\}
\end{aligned}
$$

From (2.1), (2.4) and (2.10), it follows that

$$
a^{+}(\lambda)=\lambda a_{1}+O(1), \lambda \in \overline{\mathbf{C}}_{+},|\lambda| \rightarrow \infty .
$$

Equation (3.7) shows the boundedness of set $M_{1}$ and $M_{2}$. From the analyticity of the function $a^{+}(\lambda)$ in $C_{+}$we obtain that $M_{1}$ has the most countable number of elements and its limit points can lie only in a bounded subinterval of the real axis. Using the boundary value uniqueness theorem of analytic functions, we obtain that the set $M_{2}$ is closed and $\mu\left(M_{2}\right)=0$, where $\mu\left(M_{2}\right)$ denotes the linear Lebesque measure of $M_{2}[3]$.

From (2.1) and Lemma 3.2, we get the following theorem.

Theorem 3.3. Under conditions (2.1) and (2.10), we have the following.

(i) The set of eigenvalues of the equations (2.8), (2.9) is bounded, no more than countable and its limit points can lie only in a bounded subinterval of the positive semiaxis.

(ii) The set of spectral singularities of the equations (2.8), (2.9) is bounded and its linear Lebesque measure is zero. 
Now, we recall the following.

Definition 1. The multiplicity of zero of the function $a^{+}(\lambda)$ in $\bar{C}_{+}$is defined as the multiplicity of the corresponding eigenvalue or spectral singularity of the equation (2.8), (2.9).

Theorem 3.4. If (2.10) holds and

$$
\sup _{x \in R_{+}}\left[\left|q_{k}(x)\right| \exp (\varepsilon x)\right]<\infty \quad k=1.2 \quad \varepsilon>0,
$$

then the equation (2.8), (2.9) have a finite number of eigenvalues and spectral singularities and each of them is of finite multiplicity.

Proof. From (3.8) we find that

$$
\left|H_{k j}(x, t)\right|,\left|\frac{d}{d t} H_{k j}(x, t)\right| \leq C \exp \left(-\frac{\varepsilon}{2}(x+t)\right), \quad k, j=1,2
$$

where $\mathrm{C}>0$ is a constant . (3.6),(3.9) imply that

$$
\left|S^{+}(t)\right| \leq C \exp \left(-\frac{\varepsilon}{2} t\right)
$$

It follows from (3.6) and(3.10) that the function $a^{+}(\lambda)$ has an analytic continuation from the real axis to the half plane $\operatorname{Im} \lambda-(\varepsilon / 2)$. So the limit points of the sets $M_{1}$ and $M_{2}$ can not lie in $R$, i.e., the bounded sets $M_{1}$ and $M_{2}$ have no limit points (see Lemma 3.2). Therefore, we have the finiteness of the zeros of $a^{+}(\lambda)$ in $\bar{C}_{+}$. Moreover, all zeros of $a^{+}(\lambda)$ in $\bar{C}_{+}$have finite multiplicity. Using (3.4), we obtain that the equations $(2.8),(2.9)$ have a finite number of eigenvalues and spectral singularities and each of them is of finite multiplicity.

It is seen that conditions (2.10) and (3.8) guarantee the analytic continuation of the function $a^{+}(\lambda)$ from real axis to lower half-plane. So the finiteness of eigenvalues and spectral singularities of the equations (2.8), (2.9) are obtained as a result of this analytic continuation. Now let us suppose that

$$
\sup _{x \in R_{+}}\left[\left|q_{k}(x)\right| \exp \left(\varepsilon x^{\delta}\right)\right]<\infty, k=1.2 \quad \varepsilon>0, \quad \frac{1}{2}<\delta<1,
$$

which is weaker than (3.8). It is evident that under the conditions (2.10) and (3.11), the function $a^{+}(\lambda)$ is analytic in $\bar{C}_{+}$and infinitely differentiable on the real axis. But $a^{+}(\lambda)$ does not have an analytic continuation from the real axis to lower half-plane. Therefore, under the condition (2.10), the finiteness 
of eigenvalues and spectral singularities of the equations (2.8), (2.9) can not be proved by the same technique used in theorem 3.2. Let us denote the sets of all limit points of $M_{1}$ by $M_{3}$ and the set of all zeros of $a^{+}(\lambda)$ with infinite multiplicity in $\bar{C}_{+}$by $M_{4}$. It follows from the boundary uniqueness theorem of analytic functions that

$$
M_{1} \cap M_{4}=\emptyset, \quad M_{3} \subset M_{2}, \quad M_{4} \subset M_{2},
$$

and

$$
\mu\left(M_{3}\right)=\mu\left(M_{4}\right)=0 .
$$

Using the continuity of all derivatives of $a^{+}(\lambda)$ on the real axis, we have

$$
M_{3} \subset M_{4}
$$

To prove the next result we will use the following uniqueness theorem for the analytic functions on the upper half-plane.

Theorem 3.5. (see [10].) Let us assume that the function $g$ is analytic in $\bar{C}_{+}$, all of its derivatives are continuous up to the real axis and there exists $T>0$ such that

$$
\left|g^{(n)}(z)\right| \leq C_{n}, \quad n=0,1,2, \ldots, \lambda \in C_{+}, \quad|z|<2 T,
$$

and

$$
\left|\int_{-\infty}^{-T} \frac{\ln |g(x)|}{1+x^{2}} d x\right|<\infty, \quad\left|\int_{T}^{\infty} \frac{\ln |g(x)|}{1+x^{2}} d x\right|<\infty .
$$

If the set $Q$ with linear Lebesque measure zero, is the set of all zeros of the function $g$ with infinite multiplicity and if

$$
\int_{0}^{a} \ln F(s) d \mu\left(Q_{s}\right)=-\infty
$$

then $g(z) \equiv 0$, where $F(s)=\inf _{n}\left(\frac{C_{n} s^{n}}{n !}\right), \quad n=0,1, \ldots, \mu\left(Q_{s}\right)$ is the linear Lebesque measure of s-neighborhood of $Q$ and $a$ is an arbitrary positive constant.

Lemma 3.6. If (2.10) and (3.11) hold, then $M_{4}=\emptyset$.

Proof. It follows from (3.8)-(3.10) that the function $a^{+}(\lambda)$ is analytic in $C_{+}$ and all of its derivatives are continuous up to real axis. Moreover, by Lemma 3.2. for sufficiently large $T>0$, we have

$$
\left|\int_{-\infty}^{-T} \frac{\ln \left|a^{+}(\lambda)\right|}{1+x^{2}} d x\right|<\infty, \quad\left|\int_{T}^{\infty} \frac{\ln \left|a^{+}(\lambda)\right|}{1+x^{2}} d x\right|<\infty .
$$


From (3.6), we obtain

$$
\left|a^{+}(\lambda)-\lambda a_{1}\right|<\infty, \quad \lambda \in \overline{\mathbf{C}}_{+},
$$

and

$$
\left|\frac{d^{n}}{d \lambda^{n}} a^{+}(\lambda)\right| \leq D_{n}, \quad n=1,2, \ldots
$$

where

$$
D_{1}=a_{1}+\int_{0}^{\infty} t S^{+}(t) d t, \quad D_{n}=\int_{0}^{\infty} t^{n} S^{+}(t) d t, \quad n=2,3, \ldots
$$

Using (2.4)

$$
\left|H_{k j}(x, t)\right| \leq c \exp \left[-\frac{\varepsilon}{2}\left(\frac{x+t}{2}\right)^{\delta}\right], \quad k, j=1,2, \quad \varepsilon>0, \quad \frac{1}{2}<\delta<1,
$$

and consequently,

$$
S^{+}(t) \leq c \exp \left[-\frac{\varepsilon}{2}\left(\frac{t}{2}\right)^{\delta}\right], \quad \varepsilon>0, \quad \frac{1}{2}<\delta<1,
$$

where $\mathrm{C}>0$ is a constant.From (3.17)-(3.20), we obtain

$$
\left|\frac{d^{n}}{d \lambda^{n}} a^{+}(\lambda)\right| \leq K_{n}, \quad n=1,2, \ldots, \lambda, \quad|\lambda|<2 T,
$$

where

$$
K_{n}=C \int_{0}^{\infty} t^{n} \exp \left[-\frac{\varepsilon}{2}\left(\frac{t}{2}\right)^{\delta}\right] d t
$$

It is easy to see from (3.16) and (3.21) that $a^{+}(\lambda)$ satisfies (3.14) and (3.15). Since the function $a^{+}(\lambda)$ is not equal to zero identically, then by Theorem 3.3., $M_{4}$ satisfies

$$
\int_{0}^{a} \ln F(s) d \mu\left(M_{4, s}\right)>-\infty,
$$

where $F(s)=\inf _{n}\left(\frac{K_{n} s^{n}}{n !}\right), \mu\left(M_{4, s}\right)$ is the linear Lebesque measure of sneighborhood of $M_{4}$ and the constant $K_{n}$ is defined by (3.22). Now we will obtain the following estimates for $K_{n}$ :

$$
K_{n}=C \int_{0}^{\infty} t^{n} \exp \left[-\frac{\varepsilon}{2}\left(\frac{t}{2}\right)^{\delta}\right] d t \leq B b^{n} n ! n^{n(1-\delta) / \delta},
$$


where $\mathrm{B}$ and $\mathrm{b}$ are constants depending on $\mathrm{C}, \epsilon$ and $\delta$. Substituting (3.23) in the definition of $F(s)$, we arrive at

$$
F(s)=\inf _{n}\left(\frac{K_{n} s^{n}}{n !}\right) \leq B \exp \left\{-\frac{1-\delta}{\delta} e^{-1 /(1-\delta)} b^{-\delta /(1-\delta)} s^{-\delta /(1-\delta)}\right\} .
$$

Now by (3.23), we obtain

$$
\int_{0}^{a} s^{-\delta /(1-\delta)} d \mu\left(M_{4, s}\right)<\infty .
$$

Since $\delta /(1-\delta) \geq 1,(3.25)$ holds for arbitrary s if and only if $\mu\left(M_{4, s}\right)=0$ or $M_{4}=\emptyset$.

Theorem 3.7. Under the conditions (2.10) and (3.11), The equations (2.8) and (2.9) have finite number of eigenvalues and spectral singularities, and each of them is of finite multiplicity.

Proof. To be able to prove that, we have to show that the function $a^{+}(\lambda)$ has a finite number of zeros with a finite multiplicities in $\bar{C}_{+}$. It follows from (3.12) and Lemma 3.3 that $M_{3}=\emptyset$. So the bounded set $M_{1}$ has no limit points,i.e., The function $a^{+}(\lambda)$ has finite number of zeros in $\bar{C}_{+}$. Since $M_{4}=\emptyset$, these zeros are of finite multiplicity.

\section{References}

[1] Ö. Akın and E. Bairamov, On the structure of discrete spectrum of the non-selfadjoint system of differential equations in the first order, J. Korean Math. Soc. No 3, 32, pp. 401-413, (1995).

[2] Yu. M. Berezanski, Expansion in Eigenfunctions of Selfadjoint Operators, Amer. Math., Providence R. I., (1968).

[3] E. P. Dolzhenko, Boundary Value Uniqueness Theorems for Analytic Functions, Math. Notes 25 No 6, pp. 437-442, (1979).

[4] N.B. Kerimov, A Boundary Value Problem for the Dirac System with a Spectral Parameter in the Boundary Conditions, Differential Equations, Vol. 38, No 2, pp. 164-174, (2002).

[5] E.Kır, Spectral Properties of Non-Selfadjoint System of Differential Equations, Comm. Fac.Sci. Univ. Ank. Series A1, Vol. 49, pp. 111-116, (2001).

[6] V.E.Lyance, A Differential Operator with Spectral Singularities, I,II, Amer. Math. Soc. Trans. Ser. 2, Vol. 60, pp. 227-283, (1967). 
[7] M.A.Naimark, Investigation of the Spectrum and the Expansion in Eigenfunctions of a Non-Selfadjoint Operator of Second Order on a Semi-axis, Amer. Mart. Soc. Trans. Ser. 2, Vol. 16, pp. 103-193, (1960).

[8] J.T.Schwartz, Some Non-Selfadjoint Operators, Comm. Pure and Appl. Math.13, pp. 609-939, (1960).

[9] E.Kır, G. Bascanbaz, C.Yanık, Spectral Properties of a Non Selfadjoint System of Differential Equations with a Spectral Parameter in the Boundary Condition,Universidad Catolica Del Norte, Vol. 24, pp. 49-63, (2005).

[10] E. Bairamov, O.Cakar and A. Okay Çelebi, Quadratic Pencil of Schrödinger Operators with spectral singularities: Discrete Spectrum and Principal functions, Jour. Math. Anal. Appl. 216, (1997), 303-320.

Özkan KARAMAN

Department of Mathematics,

Faculty of Science and Letters,

Kahramanmaras Sutcu Imam University,

Avsar Campus,46100, K.Maras-Turkey.

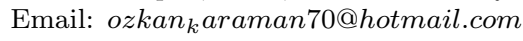


SPECTRAL PROPERTIES OF NONHOMOGENEOUS DIFFERENTIAL EQUATIONS WITH SPECTRAL PARAMETER IN THE BOUNDARY CONDITION 